\title{
Mechanism of hologram formation in fixation-free rehalogenating bleaching processes
}

\author{
Cristian Neipp, Inmaculada Pascual, and Augusto Beléndez
}

\begin{abstract}
The mechanism of hologram formation in fixation-free rehalogenating bleaching processes have been treated by different authors. The experiments carried out on Agfa 8E75 HD plates led to the conclusion that material transfer from the exposed to the unexposed zones is the main mechanism under the process. We present a simple model that explains the mechanism of hologram formation inside the emulsion. Also quantitative data obtained using both Agfa 8E75 HD and Slavich PFG-01 fine-grained red-sensitive emulsions are given and good agreement between theory and experiments are found. (C) 2002 Optical Society of America

OCIS codes: $\quad$ 090.0090, 090.2900, 090.7330, 160.2900.
\end{abstract}

\section{Introduction}

Nowadays, holography is increasingly important in different fields of optical technology, and many types of holograms are being used for scientific and technical applications. ${ }^{1}$ For example, holograms are among the most important components for the same optical systems and the physical principles of holography are being applied in fields of great importance, such as optical data storage ${ }^{2}$ or holographic optical elements. Volume phase holograms are attractive for use as optical elements or in holographic storage owing to their high-potential efficiency and high information densities. Silver halide emulsions are commonly used for recording holograms, mainly in display holography, numerous scientific applications, and in the fabrication of holographic optical elements. ${ }^{3,4}$ This is due to their high sensitivity and ease of processing, the availability of improved processing chemistries, and commercial films. Holograms recorded on silver halide emulsions are commonly bleached to improve their diffraction efficiency. Bleaching is a common method used to obtain phase holograms of high quality in photographic

C. Neipp and A. Beléndez (augusto@disc.ua.es) are with the Universidad de Alicante, Departamento de Física, Ingeniería de Sistemas y Teoría de la Señal, and I. Pascual is with the Departamento Interuniversitario de Óptica, Apartado 99, E-03080, Alicante, Spain.

Received 4 October 2001; revised manuscript received 28 January 2002.

0003-6935/02/204092-12\$15.00/0

(C) 2002 Optical Society of America emulsions. ${ }^{5}$ However, conventional bleaching techniques in which the hologram is bleached after the development and fixation steps have some disadvantages. These are, for instance, the production of high levels of scattering and the significant reduction in the thickness of the emulsion layer. Because of these reasons, processing techniques in which holograms are bleached directly after development, without a fixation step, are more adequate. In particular reversal $^{6,7}$ and fixation-free rehalogenating ${ }^{8-12}$ methods have been used during the past decades, mainly with Agfa plates. With fixation-free techniques significant changes in the average refractive index of the emulsion do not occur, ${ }^{13}$ and changes in the thickness of the emulsion layer are minimized..$^{9-14}$ The mechanism of hologram formation in fixation-free rehalogenating bleached holograms have been studied by Hariharan. ${ }^{14}$ It is assumed that diffusion of material from the exposed to the unexposed zones occurs during the bleach bath. The metallic silver grains are converted back into silver halide grains during the bleach bath by means of an oxidation process. After this oxidation process, some silver ions go into solution. Some of them are deposited in situ in the exposed zones, whereas some of them diffuse away to the unexposed zones. The refractive-index modulation is established between the exposed and nonexposed zones by the differences in size of the silver halide grains. This diffusion mechanism has been demonstrated, ${ }^{13-15}$ but a detailed theory explaining how this mechanism occurs has not been treated yet to our knowledge. Dealing with conventional bleached holograms, Van Renesse et al. ${ }^{16,17}$ presented a model that predicted how the refractive-index mod- 
Table 1. Schedule for Exposed Plates

Schedule procedure

1. Develop in $\mathrm{AAC}\left(20^{\circ} \mathrm{C}\right)$

2. Rinse in running water

3. Bleach for $1 \mathrm{~min}$ after the plate has cleared

4. Rinse in running water

5. Dry at room temperature

AAC composition

Ascorbic acid

Sodium carbonate (anhydrous)

Distilled water

$18 \mathrm{~g}$

$60 \mathrm{~g}$

11

Bleach bath composition (modified version of R-10) Solution A

Potassium dichromate

Sulfuric acid

Distilled water

Solution B

Potassium bromide

Distilled water

(Just before use, mix 1 part of A with

10 parts of distilled water,

and add 8 parts of $\mathrm{B}$ ).

ulation varies with the optical density inside the photographic emulsion. Following a similar treatment we will demonstrate that the refractive-index modulation is proportional to the optical density, and we can also derive a theoretical expression that relates the diffraction efficiency with the variations of the optical density of the hologram for fixation-free rehalogenating bleached holograms.

\section{Experimental Procedure}

Unslanted holographic transmission gratings were recorded by use of two collimated beams from a 15 $\mathrm{mW} \mathrm{He}-\mathrm{Ne}$ laser $(633 \mathrm{~nm})$, with the polarization vector prependicular to the plane of incidence. The two beams, of equal intensity, impinged on the emulsion forming an angle (in air) of $45^{\circ}$. With the geometry described, the spatial frequency of the gratings was calculated as 1200 lines $/ \mathrm{mm}$. The experiments were carried out on Agfa 8E75 HD and PFG-01 Slavich plates. After exposure, the plates underwent the scheduled procedure illustrated in Table 1 , so that rehalogenating bleached transmission holograms were finally obtained. The bleach-bath solution is composed of two different solutions: A and B. ${ }^{18,19}$ The oxidizer is contained in the solution $\mathrm{A}$, whereas the potassium bromide is contained in the solution $\mathrm{B}$. To obtain the bleach solution 1 part of $\mathrm{A}$ is mixed with 10 parts of distilled water and $X$ parts of $B$. The ratio $X=\mathrm{B} / \mathrm{A}$ indicates the relation between the potassium bromide concentration and the oxidizer concentration (potassium dichromate). The concentration of potassium bromide in the bleach bath determines the rate of the diffusion process. ${ }^{14}$ All processes described, development, washing, and bleaching were carried out at $20^{\circ} \mathrm{C}$.

Absorption holograms were also recorded by use of the geometry described. After exposure the plates were developed for $5 \mathrm{~min}$ at $20^{\circ} \mathrm{C}$ with D-19 developer. They were then washed in running water for 1 min and fixed with nonhardening F-24 fixer for 5 min at $20{ }^{\circ} \mathrm{C}$. Next, the plates were washed in running water for $10 \mathrm{~min}$ and dried in a dissecator at low humidity ( $<18 \%$ relative humidity) for $24 \mathrm{~h}$.

Measurements were made of the diffraction efficiency $\eta$ of the recorded phase holograms at the Bragg angle. The diffraction efficiency $\eta$ was calculated as the ratio of the diffracted-beam intensity to the incident collimated probe-beam intensity of the $\mathrm{He}-\mathrm{Ne}$ laser. To take into account Fresnel losses and absorption due to the glass substrate, this expression was corrected by multiplying by an appropriated factor. The losses were calculated by use of Fresnel equations to take into account the reflections that take place in the different interfaces of the hologram.

The efficiency of the zero order or transmission $\tau$ was similarly calculated as the ratio of the directly transmitted beam intensity to the incident power and was corrected by the appropriated factor to take Fresnel losses.

\section{D-LogE Curves}

To obtain the DlogE curves of Agfa 8E75 HD and Slavich PFG-01 emulsions, for D-19 developer, the amplitude holograms recorded on both emulsions were illuminated with a collimated beam from a $\mathrm{He}-\mathrm{Ne}$ laser entering normal to the surface of the plate. Measurements of the transmitted and incident light were then carried out.

The density D was obtained in terms of transmittance $\mathrm{T}$ as

$$
\mathrm{D}=\log _{10}\left(\frac{1}{\mathrm{~T}}\right)
$$

where transmittance $\mathrm{T}$ was calculated as the ratio of light transmitted through the material and light incident on the material.

The experimental data of the optical density versus the exposure were fitted with an approximation of the DlogE function obtained by Kasprzak et $\mathrm{al}^{20}$ :

$$
\mathrm{D}=\frac{\mathrm{D}_{\infty}}{1+\exp (a-b \log \mathrm{E})+\exp (c-d \log \mathrm{E})},
$$

where

$$
\begin{aligned}
& \mathrm{a}=2\left(1+\frac{2 \chi \log \mathrm{E}_{i}}{\mathrm{D}_{\infty}}\right) \\
& \mathrm{b}=\frac{4 \chi}{\mathrm{D}_{\infty}},
\end{aligned}
$$

$$
\mathrm{c}=\ln \left(\frac{\left\{\frac{\mathrm{D}_{\infty}}{\mathrm{D}_{\frac{1}{2} \log \mathrm{E}_{i}}}-\left[1+\exp \left(2+\frac{2 \chi \log \mathrm{E}_{i}}{\mathrm{D}_{\infty}}\right)\right]\right\}^{2}}{\frac{\mathrm{D}_{\infty}}{\mathrm{D}\left(\log \mathrm{E}_{i}\right)}-\left(1+\mathrm{e}^{2}\right)}\right)^{2}
$$




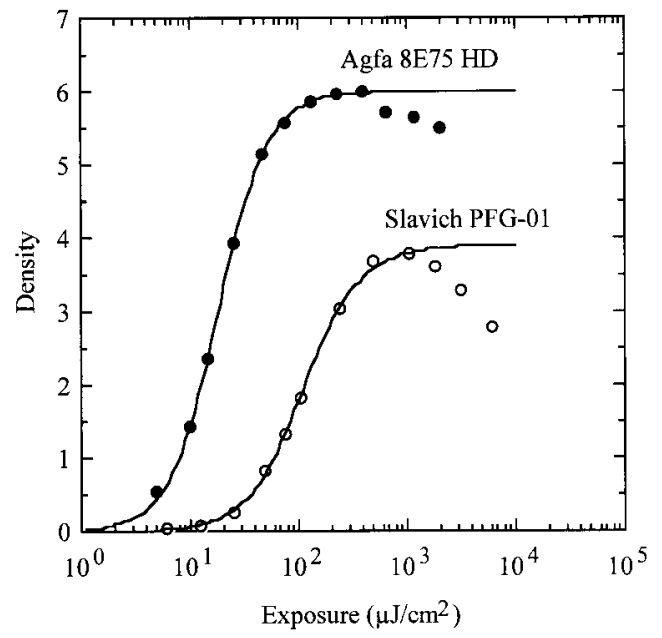

Fig. 1. DlogE curves for absorption holograms recorded on Agfa 8E75 HD and Slavich PFG-01 plates.

$$
\mathrm{d}=\frac{\mathrm{c}-\ln \left[\frac{\mathrm{D}_{\infty}}{\mathrm{D}\left(\log \mathrm{E}_{i}\right)}-\left(1+\mathrm{e}^{2}\right)\right]}{\log \mathrm{E}_{i}} .
$$

$\chi$ is the slope of the characteristic curve in the linear region. $\mathrm{E}_{i}$ is the exposure of the inertial point, and $\mathrm{D}_{\infty}$ is the maximum of the measured optical density. $\mathrm{E}_{i}$ gives information about the energetic sensitivity of the particular emulsion. The lower the value of $\mathrm{E}_{i}$ the higher the energetic sensitivity of the emulsion.

Figure 1 shows the DlogE curves for Slavich PFG-01 and Agfa 8E75 HD plates and the theoretical curves obtained by use of the Kasprzak equation. There's good agreement between theory and experiment for both Agfa and Slavich plates below the solarization region, this region is not well described in the case of the Slavich PFG-01 plates.

Table 2 shows the values of $\chi, \mathrm{D}_{\infty}$, and $\mathrm{E}_{i}$ for both Agfa and Slavich plates. The energetic sensitivity presented by Agfa 8E75 HD plates is higher than that of Slavich PFG-01 plates, and also the maximum density obtained with Agfa plates is higher. The reason for these differences is the concentration of silver halide grains suspended in the gelatin of both emulsions. The concentration of silver halide grains inside the emulsion can be calculated according to the following expression ${ }^{3,21}$ :

$$
N=\frac{6 m_{\mathrm{AgBr}}}{d \pi \delta^{3} \rho},
$$

where $\rho$ is the density of the silver bromide $(6.47$ $\left.\mathrm{g} / \mathrm{cm}^{3}\right), \mathrm{m}_{\mathrm{AgBr}}$ is the mass per unit surface of silver

Table 2. Energetic Sensitivity Values

\begin{tabular}{lccc}
\hline \multicolumn{1}{c}{ Emulsion } & $\mathrm{D}_{\infty}$ & $\chi$ & $\mathrm{E}_{i}\left(\mu \mathrm{J} / \mathrm{cm}^{2}\right)$ \\
\hline Agfa 8E75 HD & 6.0 & 6.9 & 5.3 \\
PFG-01 de Slavich & 3.8 & 3.3 & 28 \\
\hline
\end{tabular}

Table 3. Values Given by Manufacturer

\begin{tabular}{lccc}
\hline \multicolumn{1}{c}{ Emulsion } & $\delta(\mathrm{nm})$ & $m_{\mathrm{AgBr}}\left(\mathrm{g} / \mathrm{m}^{2}\right)$ & $N\left(\right.$ grains $\left./ \mathrm{cm}^{3}\right)$ \\
\hline Agfa 8E75 HD & 44 & 8.7 & $5.2 \times 10^{15}$ \\
PFG-01 & 40 & 2.7 & $1.7 \times 10^{15}$ \\
\hline
\end{tabular}

halide, $\delta$ is the mean grain size of a silver halide grain inside the emulsion, and $d$ is the thickness of the emulsion. The values of the thickness of the layer, $d$, for Agfa 8E75 HD and PFG-01 emulsions were measured experimentally with a procedure based on the film-resonance method reported by Thöll et al., ${ }^{22}$ obtaining $d \sim 6 \mu \mathrm{m}$ and $d \sim 7 \mu \mathrm{m}$ for Agfa 8E75 HD and PFG-01 emulsions, respectively. The values of $\mathrm{m}_{\mathrm{AgBr}}$ and $\delta$, given by the manufacturer, and the values of $N$, obtained by Eq. (7), are illustrated in Table 3 for both emulsions. It can be seen that whereas the value of $\delta$ is similar for both emulsions, $\delta \sim 40 \mathrm{~nm}$, the concentration of silver halide grains is higher in the case of the Agfa 8E75 HD emulsion. Owing to the lower concentration of silver halide grains in PFG-01 plates, the energetic sensitivity and the maximum optical density are lower than in the case of Agfa 8E75 HD plates.

\section{Theoretical Model and Experimental Results}

To obtain an hologram in a photographic emulsion, the plate is exposed, developed, and bleached.

\section{A. Exposure and Development}

After the exposure and development the variations of intensity created by the interference pattern are converted in optical-density differences inside the hologram.

The optical density of an exposed zone can be related to the partial atomic concentration of silver inside the emulsion, $N_{\mathrm{s}}{ }^{17}$ :

$$
\mathrm{D}=\frac{a N_{\mathrm{s}} d_{r}}{\cos \theta^{\prime}}
$$

$d_{r}$ is the thickness of the emulsion after the development step, $\theta^{\prime}$ is the angle that light forms with the normal to the plate inside the emulsion and $a$ is an absorption constant. ${ }^{17}$

We define the following parameters: $N^{0}$ the initial concentration of silver halide molecules inside the photographic emulsion. $\quad N_{d}^{1}$ concentration of silver halide molecules remaining inside the emulsion after the exposure and development step, and $N_{d}{ }^{2}$ concentration of developed silver halide molecules, namely the silver halide molecules that have been converted into silver atoms (Fig. 2).

The concentration of silver atoms after development, $N_{\mathrm{s}}$, can be related to the concentration of silver halide molecules that have been developed through $q_{d}$, the number of silver halide molecules that yield to one silver atom:

$$
N_{\mathrm{s}}=\frac{N_{d}^{2}}{q_{d}}=\frac{N^{0}-N_{d}^{1}}{q_{d}} .
$$


exposure+development

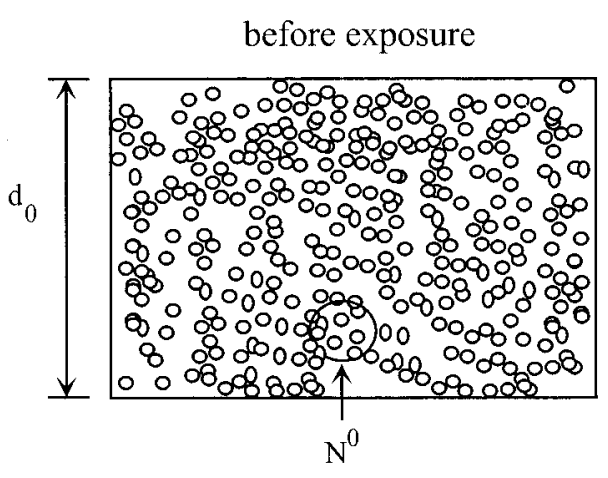

Fig. 2. Two zones of the emulsion before and after the exposure and development.

The swelling factor after the development step is defined as the ratio of the emulsion thickness after the development step, $d_{r}$, and the thickness before the development step, $d_{0}$ :

$$
m_{r}=\frac{d_{r}}{d_{0}}
$$

\section{B. Bleaching Step}

The aim of the bleaching bath is to convert the metallic silver back into silver halide. This action takes place through an oxidation reaction. For instance, in the case of dichromate bleaches, the following oxidation reaction occurs:

$$
6 \mathrm{Ag}+\mathrm{Cr}_{2} \mathrm{O}_{7}^{-2}+14 \mathrm{H}^{+} \rightarrow 6 \mathrm{Ag}^{+}+\mathrm{Cr}^{+3}+7 \mathrm{H}_{2} \mathrm{O}
$$

The silver ions $\mathrm{Ag}^{+}$combine with the negative ions of the halide atom provided by the rehalogenant agent. For example, when the rehalogenant agent is potassium bromide:

$$
\mathrm{Ag}^{+}+\mathrm{KB}_{\mathrm{r}} \rightarrow \mathrm{Ag} \mathrm{B} \mathrm{B}_{\mathrm{r}}+\mathrm{K}^{-}
$$

If $q_{b}$ quantifies the number of silver atoms yielding to a silver halide molecule formed through the oxidation reaction, the concentration of rehalogenated silver halide molecules that are formed after the bleaching bath, $N_{\mathrm{b}}$ is

$$
N_{\mathrm{b}}=\frac{N_{\mathrm{s}}}{q_{\mathrm{b}}} .
$$

The mechanism by which the metallic silver is rehalogenated to silver bromide is more complex than it might seem at first sight. Hariharan suggested that the $\mathrm{Ag}^{+}$ions of reaction (12) go into solution, some of them are redeposited in situ in the exposed zones, whereas others diffuse away to the unexposed zones contributing to an increase in the grain size of the silver bromide grains in these zones. The refractiveindex modulation is a consequence of the differences in the grain size of the silver halide grains in the exposed and non-exposed zones. This is the basis of the fixation-free bleaching techniques. They are called fixation-free because the fixing step included in the conventional bleaching techniques is eliminated from the procedure. The action of the fixing bath is to remove the unexposed silver halide grains from the emulsion, but as we have explained in the fixationfree rehalogenating technique this is not necessary.

The diffusion mechanism can be explained from the differences in concentration of the silver atoms between the exposed and non-exposed zones. Let us consider two zones of emulsion presenting a variation of the optical density. Zone 1 presents a higher optical density than zone 2. This implies a higher concentration of silver atoms in zone 1 and a consequent higher concentration of silver ions, $N_{\mathrm{si}}$ coming from the oxidation reaction in zone 1 :

$$
N_{\mathrm{s} 1}>N_{\mathrm{s} 2} \Rightarrow N_{\mathrm{si} 1}>N_{\mathrm{si} 2} \text {. }
$$

These differences in concentration create a diffusion process of silver ions from zone 1 to zone 2 , in a similar way as the Fick molecular diffusion mechanism. Nevertheless, to simplify the mathematical treatment, it will be assumed that the number of silver ions going from zone 1 to zone $2, N_{\text {sid }}$ is proportional to the difference in concentration $N_{\mathrm{si1}}-$ $N_{\mathrm{si} 2}$ and to the time in which the plates are maintained in the bleaching bath:

$$
N_{\mathrm{sid}}=\gamma t\left(N_{\mathrm{si} 1}-N_{\mathrm{si} 2}\right) .
$$

Here the parameter $\gamma$ gives an idea of the rate of the diffusion mechanism. It is influenced by factors, such as the B/A, as will be demonstrated in Subsection 4. E, the degree of hardening of the gelatin, the proper chemical compounds of the gelatin, etc. Another factor influencing parameter $\gamma$ is the spatial frequency, which as commented on by Hariharan ${ }^{23}$ influences the rate of the diffusion process, an influence which is related with the mean life of the silver ion.

Because the number of silver ions is proportional through $q_{\mathrm{b}}$ to the number of rehalogenated silver halide molecules, the concentration of rehalogen- 


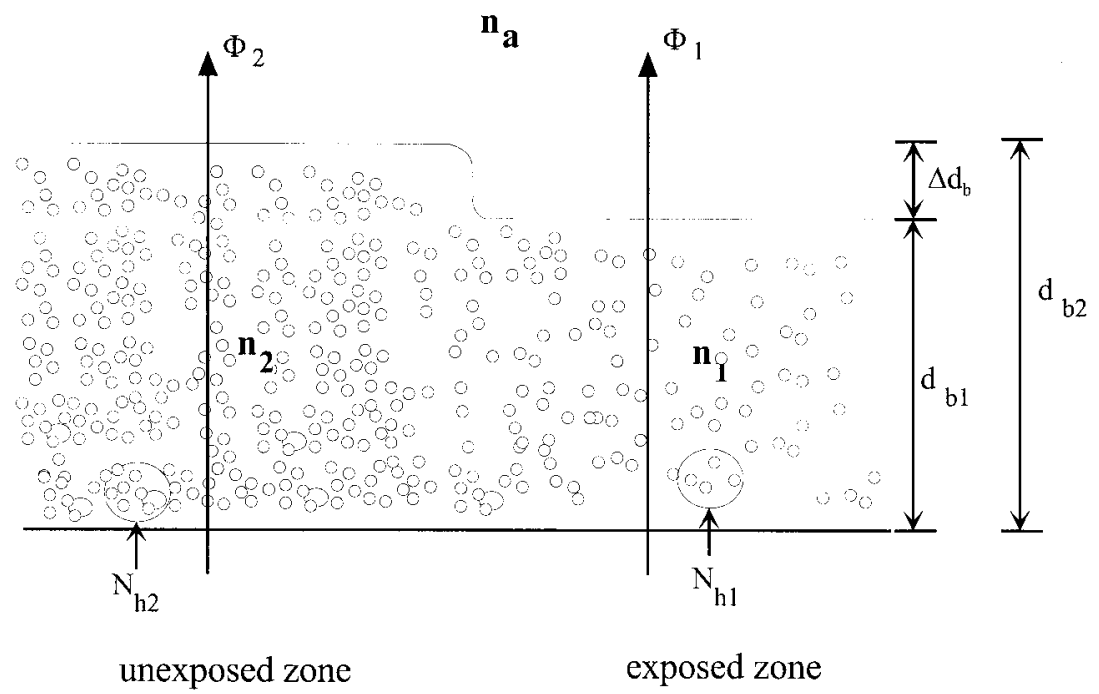

Fig. 3. Two zones of the emulsion after the bleach bath.

ated silver halide molecules in zone 1 will be $N_{\mathrm{b} 1}-$ $\gamma t\left(N_{\mathrm{b} 1}-N_{\mathrm{b} 2}\right)$, whereas in zone $2, N_{\mathrm{b} 2}+\gamma t\left(N_{\mathrm{b} 1}-\right.$ $N_{\mathrm{b} 2}$ ), where

$$
N_{b}=\frac{N_{\mathrm{si}}}{\mathrm{q}_{\mathrm{b}}} .
$$

The total concentration of silver halide molecules in zones 1 and $2, N_{\mathrm{h} 1}$ and $N_{\mathrm{h} 2}$, respectively, will be:

$$
\begin{aligned}
& N_{\mathrm{h} 1}=N_{d 1}{ }^{1}+N_{\mathrm{b} 1}-\gamma t\left(N_{\mathrm{b} 1}-N_{\mathrm{b} 2}\right) \\
& N_{\mathrm{h} 2}=N_{d 2}{ }^{1}+N_{\mathrm{b} 2}+\gamma t\left(N_{\mathrm{b} 1}-N_{\mathrm{b} 2}\right) .
\end{aligned}
$$

The swelling factor after the bleaching bath is defined as

$$
m_{\mathrm{b}}=\frac{d_{\mathrm{b}}}{d_{0}}
$$

$d_{\mathrm{b}}$ is the thickness after the bleaching bath

The swelling factor between the development step and the bleaching step can be related through m:

$$
m=\frac{d_{\mathrm{b}}}{d_{r}}=\frac{m_{\mathrm{b}}}{m_{r}}
$$

\section{Phase Difference as a Function of the Variation in Optical Density}

As explained, owing to the bleaching process without a fixation step, differences in the emulsion appear in the concentration of the silver halide molecules between the exposed and non-exposed zones. Because of these differences in concentration, the refractive index in the exposed zones is different than the refractive index in the non-exposed zones.

The phase difference between two beams of light going through two different zones of the emulsion with different concentrations of silver halide molecules (Fig. 3) can be obtained according to the follow- ing equation ${ }^{17}$ :

$$
\Phi_{2}-\Phi_{1}=\frac{2 \pi\left(d_{\mathrm{b} 2} n_{2}-d_{\mathrm{b} 1} n_{1}-\Delta d_{\mathrm{b}} n_{\mathrm{a}}\right)}{\lambda \cos \theta^{\prime}} .
$$

$n_{1}$ and $n_{2}$ are the refractive indexes of zones 1 and 2 , respectively, $n_{\mathrm{a}}$ is the refractive index of air, $\theta^{\prime}$ is the angle that the incident light forms with the normal of the emulsion, $d_{\mathrm{b} 1}$ and $d_{\mathrm{b} 2}$ are the emulsion thickness of zones 1 and 2 , respectively, $\Delta d_{\mathrm{b}}$ is the thickness variation between the two zones, and $\lambda$ is the wavelength of light in air.

In this analysis the fine grained photographic emulsion is considered as an homogenous mixture with Rayleigh scatter centers. In this case, the Lorentz-Lorenz equation can be applied to obtain the refractive index of a mixture of substances. We used the linear approximation of the LorentzLorenz equation given by van Renesse et al. ${ }^{17}$ :

$$
n=\mathrm{c} \sum_{i} \alpha_{i} N_{i}+n_{0}
$$

$\alpha_{i}$ is the electrical polarizability of molecules of type $i$ in the mixture and $N_{i}$ the concentration of molecules of type $i$ in the mixture. The values of $\mathrm{c}$ and $n_{0}$ are: $\mathrm{c}=8.7$ y $n_{0}=0.89$.

By use of Eqs. (8)-(10) and Eqs. (17)-(22) the phase difference between the two waves passing through the two zones of the emulsions can be expressed in the following form:

$$
\begin{aligned}
\Phi_{2}-\Phi_{1}= & \frac{2 \pi}{\lambda a} \operatorname{c} \alpha_{\mathrm{h}}\left[m_{2} \mathrm{D}_{2}\left(\frac{1-\gamma t-\gamma t / L}{q_{\mathrm{b}}}-q_{r}\right)\right. \\
& \left.-m_{1} \mathrm{D}_{1}\left(\frac{1-\gamma t-\gamma t / L}{q_{\mathrm{b}}}-q_{\mathrm{r}}\right)\right]+ \\
& +\frac{2 \pi}{\lambda \cos \theta^{\prime}} \Delta d_{\mathrm{b}}\left(n_{0}-n_{\mathrm{a}}+\mathrm{c} \alpha_{\mathrm{h}} N^{0}\right),
\end{aligned}
$$


where

$$
L=\frac{d_{\mathrm{b} 2}}{d_{\mathrm{b} 1}}
$$

The second term of Eq. (23) expresses the phase modulation due to the thickness modulation. This term must be considered when recording low spatial frequency holograms, but not in the case of high spatial frequencies. If the thickness modulation is not considered, the following relations are also valid:

$$
\begin{aligned}
L & =1, \\
m_{2} & =m_{1}=m=\frac{m_{\mathrm{b}}}{m_{r}} .
\end{aligned}
$$

In these conditions the phase difference becomes

$$
\Phi_{2}-\Phi_{1}=\frac{2 \pi}{\lambda \mathrm{a}} \mathrm{c} \alpha_{\mathrm{h}} m\left(\frac{1-2 \gamma t}{q_{\mathrm{b}}}-q_{r}\right)\left(\mathrm{D}_{2}-\mathrm{D}_{1}\right)
$$

This relation indicates that the phase difference is proportional to the optical density variations inside the emulsion.

From each $\mathrm{Ag} \mathrm{Br}$ molecule, one silver atom is obtained after the exposure and development, therefore $q_{r}=1$. According to reaction (12), if a silver bromide molecule is formed from each silver atom, then $q_{\mathrm{b}}=$ 1. In this condition:

$$
\Delta \Phi=-\frac{4 \pi}{\lambda \mathrm{a}} \mathrm{c} \alpha_{\mathrm{h}} m \gamma t \Delta \mathrm{D} .
$$

The minus sign indicates that the index modulation created through the diffusion mechanism is established from zones of higher optical density to zones of lower optical densities.

\section{Diffraction Efficiency as a Function of the} Optical Density

For sinusoidal gratings the diffraction efficiency is related to the phase difference through the following equation:

$$
\eta=\sin ^{2}\left(\frac{\Delta \Phi}{4}\right),
$$

where $\Delta \Phi$ is the maximum phase difference between two zones of the emulsion. By use of Eq. (28) a relation between the diffraction efficiency and the maximum optical density store in the hologram, $\Delta \mathrm{D}$, can be made:

$$
\eta=\sin ^{2}\left(\frac{\pi}{\lambda \mathrm{a}} \mathrm{c} \alpha_{\mathrm{h}} m \gamma t \Delta \mathrm{D}\right) .
$$

In this work we have recorded unslanted transmission holographic gratings with an intensity ratio $K=$ $1, K=I_{\mathrm{o}} / I_{\mathrm{r}}$, where $I_{r}$ is the intensity of the reference wave and $I_{o}$ is the intensity of the object wave. The theoretical visibility is

$$
V=\frac{2 \sqrt{I_{r} I_{o}}}{I_{r}+I_{o}}=1 .
$$

The optical density variation between a zone exposed to the maximum intensity of the light and a nonexposed zone is, therefore:

$$
\Delta \mathrm{D}=\mathrm{D}_{\max }-\mathrm{D}_{\min }=\mathrm{D}_{\max }-0 .
$$

where $\mathrm{D}_{\max }$ is the maximum store optical density, whereas the minimum optical density store $\mathrm{D}_{\min }$ is 0 . Nevertheless, $D_{\min }$ is non zero because of several factors: scattering in the recording, limited modulation transfer function of the material, adjacent effects, etc.

The maximum optical densities created inside the emulsion should then be expressed as:

$$
\Delta \mathrm{D}=\mathrm{D}-\mathrm{D}_{0}
$$

$\mathrm{D}_{0}$ is the minimum store optical density in the nonexposed zones and $\mathrm{D}$ is the optical density to which the holograms are exposed.

The diffraction efficiency can be expressed as follows:

$$
\eta=\sin ^{2}\left[\frac{\pi}{\lambda \mathrm{a}} \operatorname{co} \alpha_{\mathrm{h}} m \gamma t\left(\mathrm{D}-\mathrm{D}_{0}\right)\right] .
$$

E. Fit of the Theoretical Function of the Diffraction Efficiency as a Function of the Optical Density

To test the proposed model the function of the diffraction efficiency versus the optical density was fitted for holographic gratings recorded on Agfa 8E75 HD plates.

The diffraction efficiency was measured as a function of the exposure. By use of the fitting to the Kaszprak curve of the experimental DlogE curves, the response of the diffraction efficiency as a function of the optical density can be obtained.

In expression (34) the absorption and scattering (A\&S) of the holograms has not been taken into account. The diffraction efficiency must be corrected to take into account the A\&S losses. Figure 4 shows the values of the A\&S losses as a function of the exposure for holographic gratings recorded on Agfa 8E75 HD emulsion and developed with D-19 developer. The A\&S was calculated as $1-\eta-\tau$. It can be seen that A\&S coefficient depends linearly with the optical density in the range of exposures studied. This fact allows a linear fitting to the experimental data. The continuous lines correspond to the theoretical fitting, supposing that the A\&S depends on the optical density according to

$$
\mathrm{A} \& \mathrm{~S}=m \mathrm{D}+n .
$$

The values of $m$ and $n$ are shown in Table 4 .

If the dependence of the A\&S coefficient with the 

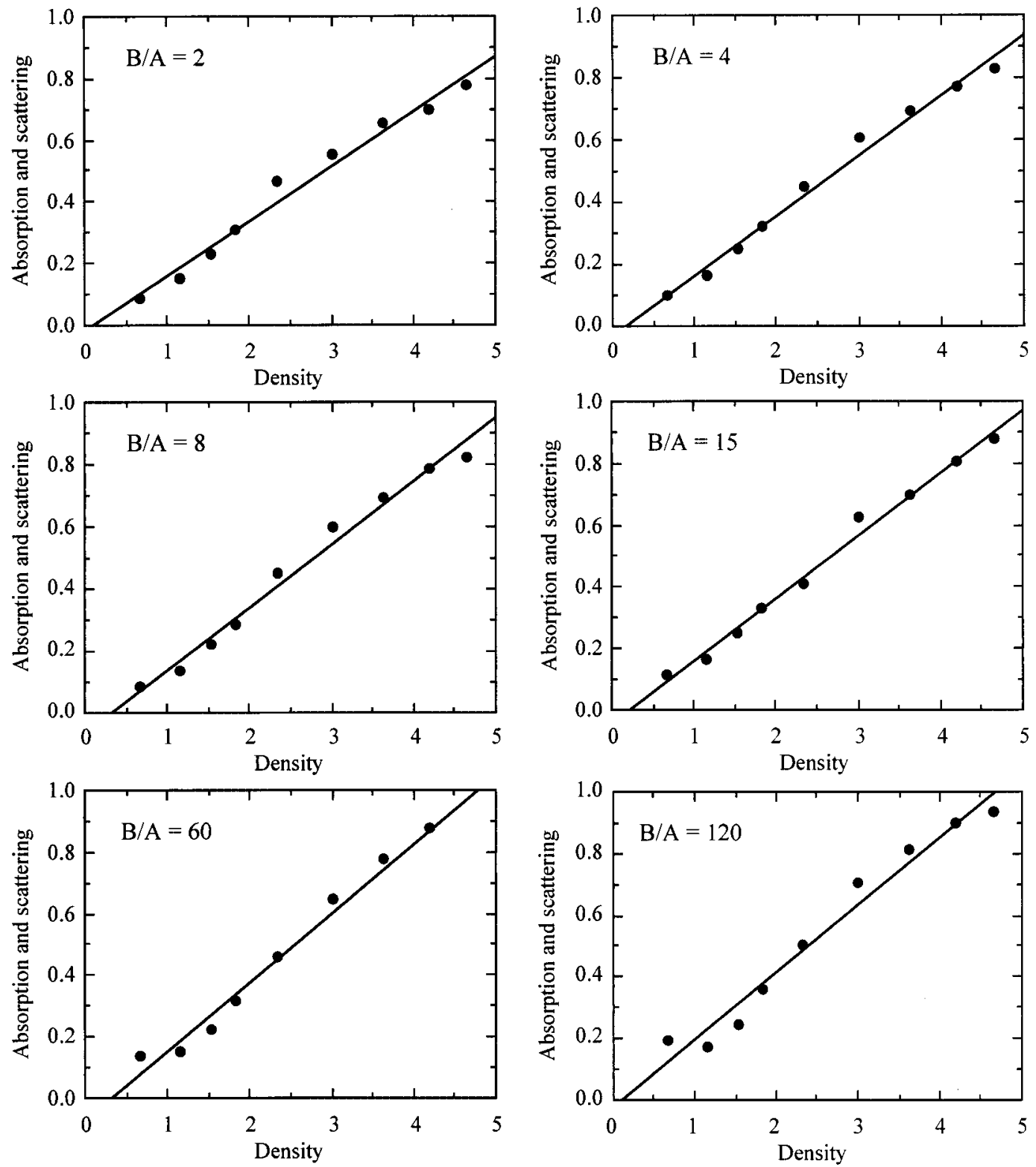

Fig. 4. Absorption and scattering as a function of the optical density for transmission diffraction gratings recorded on Agfa 8E75 HD emulsion.

optical density is known, the diffraction efficiency as a function of the optical density has the form:

$$
\eta=f(1-A \& S)\left(\sin ^{2}\left[h\left(D-D_{0}\right)\right]\right.
$$

Table 4. Values of $m$ and $n$

\begin{tabular}{rcc}
\hline $\mathrm{B} / \mathrm{A}$ & $m$ & $n$ \\
\hline 2 & 0.1788 & -0.0202 \\
4 & 0.1929 & -0.0272 \\
8 & 0.2016 & -0.0613 \\
15 & 0.2026 & -0.0423 \\
60 & 0.2237 & -0.0693 \\
120 & 0.2188 & -0.0562 \\
\hline
\end{tabular}

where

$$
\mathrm{h}=\frac{\pi}{\lambda \mathrm{a}} \mathrm{c} \alpha_{\mathrm{h}} m \gamma t
$$

and $\mathrm{f}$ is an empirical factor.

Figure 5 shows the diffraction efficiency as a function of the optical density for the holographic diffraction gratings recorded on Agfa 8E75 HD emulsion and developed with D-19 developer, whereas Table 5 illustrates the values of $h, D_{0}$ and $f$ for the different $\mathrm{B} / \mathrm{A}$ ratios.

Good agreement between theory and experiment can be found. The value of $\mathrm{D}_{0}$ is maintained almost constant, $\mathrm{D}_{0} \sim 1$, indicating that this value does not depend on the $\mathrm{B} / \mathrm{A}$ ratio. 

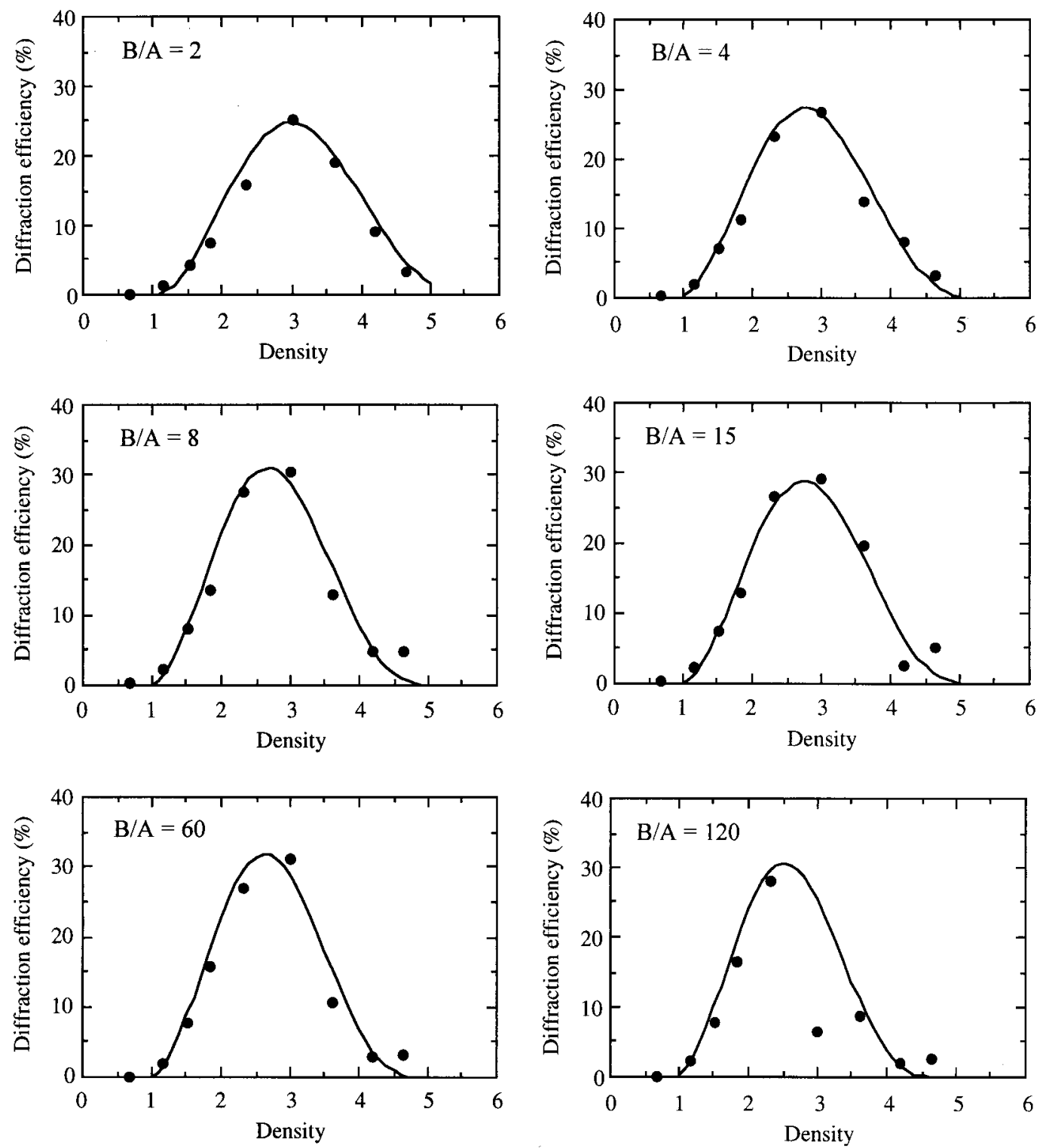

Fig. 5. Diffraction efficiency as a function of the optical density for transmission diffraction gratings recorded on Agfa 8E75 HD emulsion.

F. Refractive Index Modulation as a Function of the Optical Density

By use of Eqs. (8), (9), (17), (18), and (22) an expression of the refractive index modulation as a function of the optical density can also be obtained:

$$
\Delta n=-\frac{2 \mathrm{c} \alpha_{\mathrm{h}} \gamma t \cos \theta^{\prime} \Delta \mathrm{D}}{\mathrm{a} d_{r}} .
$$

Table 5. Values for Different B/A Ratios

\begin{tabular}{rccc}
\hline $\mathrm{B} / \mathrm{A}$ & $\mathrm{h}$ & $\mathrm{D}_{0}$ & $\mathrm{f}$ \\
\hline 2 & 0.6618 & 1.0244 & 0.55 \\
4 & 0.6926 & 0.9103 & 0.62 \\
8 & 0.7561 & 0.9435 & 0.65 \\
15 & 0.7107 & 0.9521 & 0.65 \\
60 & 0.7628 & 0.9572 & 0.72 \\
120 & 0.8069 & 0.9185 & 0.72 \\
\hline
\end{tabular}

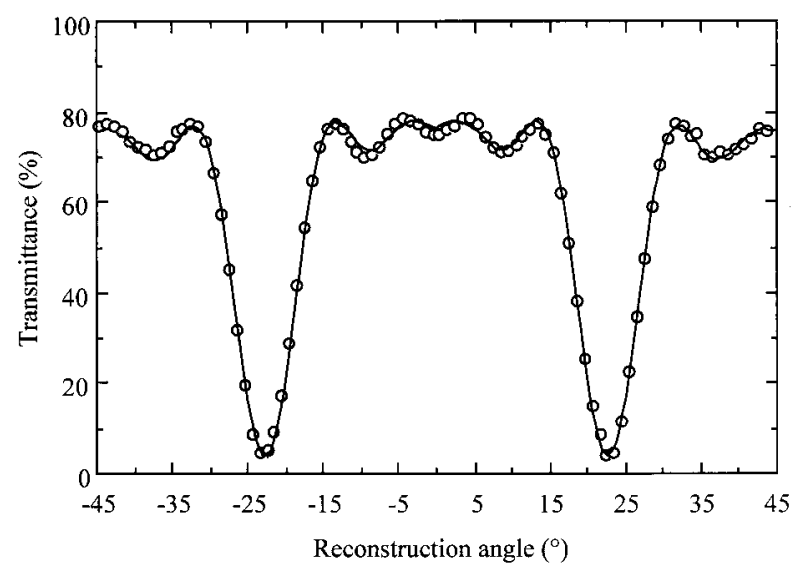

Fig. 6. Transmittance as a function of the angle of reconstruction for transmission diffraction gratings recorded on PFG-01 emulsion developed with D-19 and bleached with a B/A ratio of 60 . 
Table 6. Theoretical Fitting Values for Agfa 8E75 HD

\begin{tabular}{rcc}
\hline $\mathrm{B} / \mathrm{A}$ & $\omega$ & $\mathrm{D}_{0}$ \\
\hline 2 & 0.0164 & 1.0122 \\
4 & 0.0173 & 1.0116 \\
8 & 0.0171 & 0.9298 \\
15 & 0.0191 & 1.0367 \\
60 & 0.0207 & 1.0290 \\
120 & 0.0230 & 1.0387 \\
\hline
\end{tabular}

For unslanted transmission holographic gratings with a beam ratio $1: 1, \Delta n$ has the form:

$$
\Delta n=\omega\left(\mathrm{D}-\mathrm{D}_{0}\right)
$$

where

$$
\omega=\frac{2 \operatorname{co} \alpha_{\mathrm{h}} \gamma t \cos \theta^{\prime}}{\mathrm{a} d_{r}}
$$

c, $\alpha_{h}$, and a are constants for all the holographic gratings recorded on a same emulsion. Whereas $d_{r}$ depends on the initial thickness of the emulsion and of the swelling factor during the development step, which mainly depends on the developer used and on the bleach-bath temperature. For holographic gratings recorded on the same emulsion and developed in the same conditions, the swelling factor must be considered the same. In those conditions the growth of the index modulation with the optical density is related with the rehalogenating bleaching time, $t$, and the parameter $\gamma$. The $\gamma$ parameter indicates the
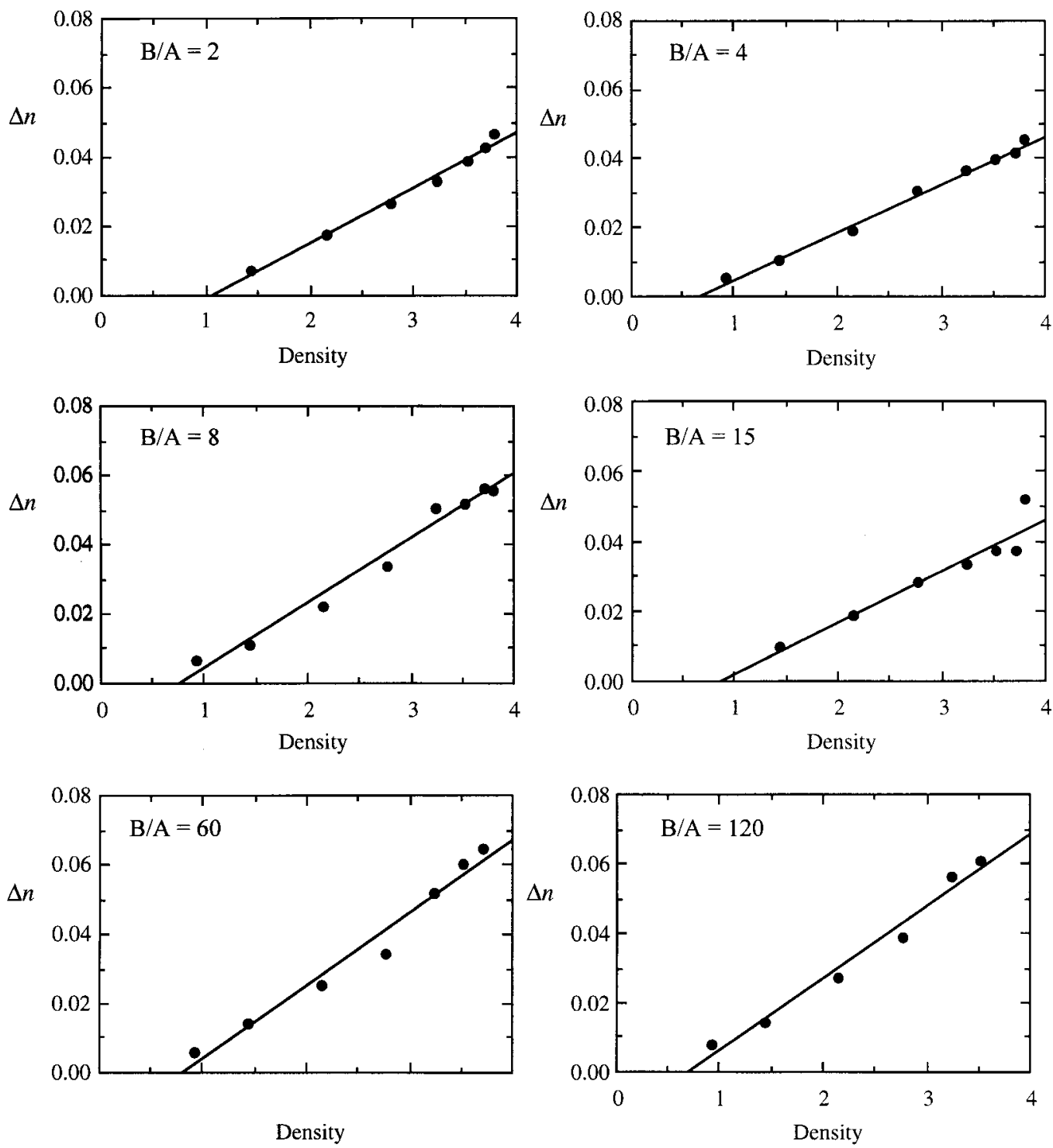

Fig. 7. Refractive index modulation, $n$, as a function of the optical density for transmission diffraction gratings recorded on Agfa $8 \mathrm{E} 75$ HD emulsion. 

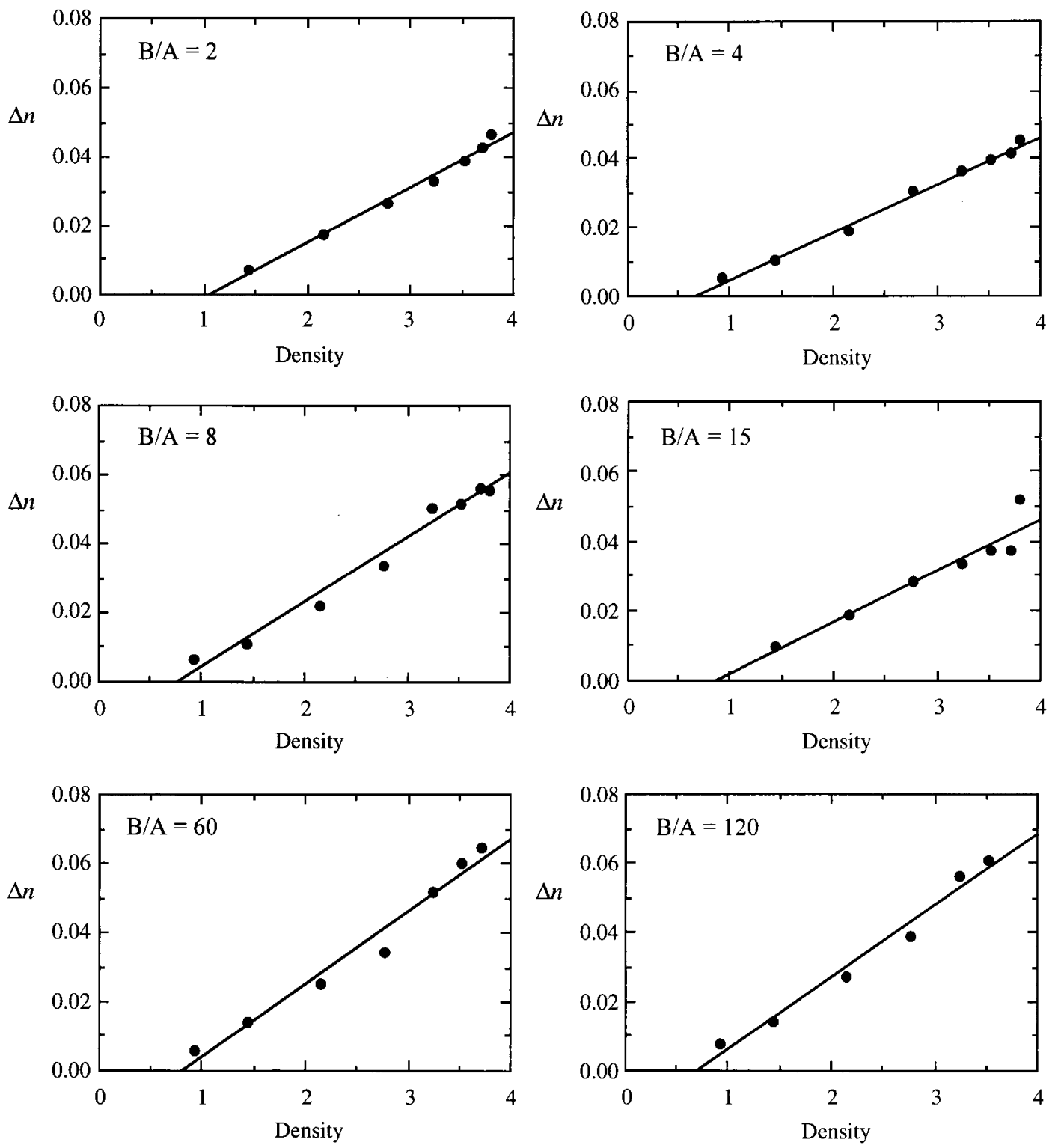

Fig. 8. Refractive index modulation, $n$, as a function of the optical density for transmission diffraction gratings recorded on PFG-01 emulsion.

rate of the diffusion process of the silver ion from the exposed to the non-exposed zones.

To obtain the values of the refractive index modulation, $\Delta n$, we tested the holograms by rotating them, and the variation in transmission with the angle of incidence $\tau$ in air was measured. The rotation axes stayed in the plane of the hologram and perpendicular to the plane of incidence. The values of transmission were corrected to take into account Fresnel's reflections and the absorption of the glass substrate. By using Kogelnik coupled wave theory ${ }^{24}$ the theoretical function of the transmittance was fitted to the experimental data, so we obtained information about the refractive index modulation, the thickness, and the absorption coefficient. Figure 6 shows the transmission efficiency as a function of the reconstruction angle for a bleached transmission grating recorded on
PFG-01 emulsion developed with D-19 and bleached with a $\mathrm{B} / \mathrm{A}$ ratio of 60 . Good agreement between the theoretical function and the experimental data can be seen.

Figure 7 shows the refractive index modulation as a function of the optical density for transmission holographic gratings developed with D-19 and recorded on Agfa 8E75 HD emulsion and bleached with different $\mathrm{B} / \mathrm{A}$ ratios. Figure 8 shows the refractive index values as a function of the optical density for holographic gratings developed with D-19 and store on PFG-01 emulsion. In both figures the continuous lines correspond to the theoretical fitting with Eq. (38).

Tables 6 and 7 illustrate the values obtained by the theoretical fittings of the studied gratings. The values of Table 6 correspond to gratings recorded on 
Table 7. Theoretical Fitting Values for Slavich 8E75-PFG01

\begin{tabular}{rcc}
\hline $\mathrm{B} / \mathrm{A}$ & $\omega$ & $\mathrm{D}_{0}$ \\
\hline 2 & 0.0159 & 1.0566 \\
4 & 0.0139 & 0.6403 \\
8 & 0.0186 & 0.7688 \\
15 & 0.0148 & 0.8649 \\
60 & 0.0209 & 0.7943 \\
120 & 0.0206 & 0.6845 \\
\hline
\end{tabular}

Agfa 8E75 HD emulsion, whereas the values of Table 7 correspond to gratings recorded on Slavich PFG-01 emulsion. The value of $D_{0}$ obtained in the case of the holographic gratings recorded on the Agfa 8E75 $\mathrm{HD}$ emulsion is almost the same for all the cases and coincides with the value of $\mathrm{D}_{0}$ calculated in Subsection 4.D.

Figures 9 and 10 show the value of the slopes of the regression lines of Figs 7 and 8 as a function of the $\mathrm{B} / \mathrm{A}$ ratio for Agfa and Slavich plates, respectively. It can be seen that in the case of Agfa 8E75 HD plates the slope of the regression line increases as does the

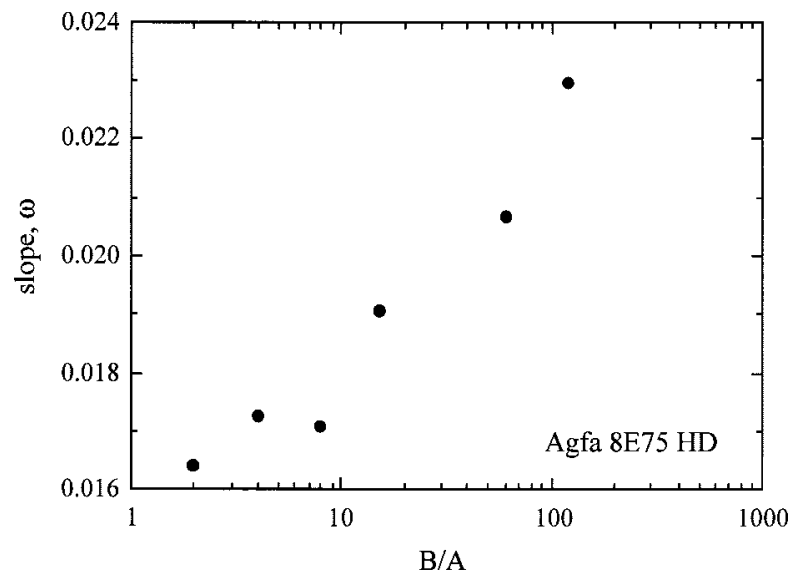

Fig. 9. Slopes, $\omega$, of the regression lines of Fig. 4 as a function of the $\mathrm{B} / \mathrm{A}$ ratio.

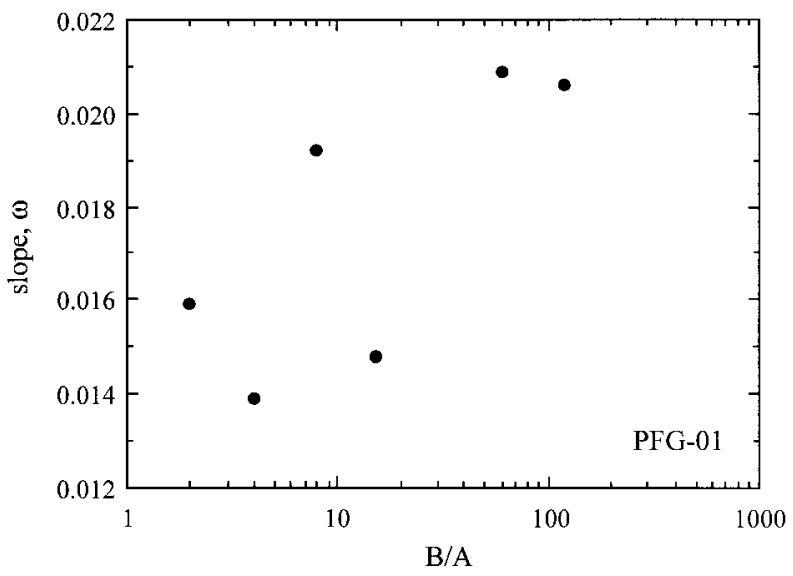

Fig. 10. Slopes, $\omega$, of the regression lines of Fig. 5 as a function of the $\mathrm{B} / \mathrm{A}$ ratio.
$\mathrm{B} / \mathrm{A}$ ratio. The reason for this behavior is that as the $\mathrm{B} / \mathrm{A}$ ratio increases, so does the bleaching time, but as indicated by Hariharan, the solubility of silver ions is higher for higher $\mathrm{B} / \mathrm{A}$ ratios, namely, higher potassium bromide concentrations. Therefore the value of $\gamma$ grows with the $\mathrm{B} / \mathrm{A}$ ratio and so does the slope of Eq. (38), $\omega$. With respect to the diffraction gratings recorded on Slavich PFG-01 emulsion, the same tendency is observed. Nevertheless, there are deviations from the expected behavior. The degree of hardening of the gelatin also influences the diffusion process, because a high degree of hardening limits the diffusion of the silver ion. Because Slavich PFG-01 gelatin is very soft, the hardening effects of $\mathrm{Cr}^{+3}$ [after reaction (11) $]^{11}$ seem to be, then, more critical than in the case of Agfa 8E75 HD emulsion, which has a harder gelatin. These effects affect the diffusion process and slightly alter the behavior observed with Agfa 8E75 HD plates.

\section{Conclusions}

The mechanism of hologram formation in fixationfree rehalogenating bleaches has been explained. It is assumed that there is a diffusion of silver ions from the exposed to the unexposed zones in the bleach bath. Under this assumption it has been demonstrated that in the case of unslanted phase transmission gratings the phase difference between two zones of the emulsion depends linearly on the optical density variations. The experiments carried out with Agfa 8E75 HD plates confirm the theoretical model. An expression of the refractive index modulation as a function of the optical density has also been obtained, and we find that this is also proportional to the optical density variations. From the experiments carried out with Agfa 8E75 HD and Slavich PFG-01 plates it was demonstrated that the slope of the linear line representing the index modulation as a function of the optical density increases with the $\mathrm{B} / \mathrm{A}$ ratio.

This work was financed by the CICYT (Comisión Interministerial de Ciencia y Tecnología, Spain) under project No. MAT2000-1361-C04-04. We thank I. Arias for her interest in this work and for her helpful discussion.

\section{References}

1. H. I. Bjelkhagen and H. J. Caulfield, eds. Selected Papers on Fundamental Techniques in Holography, SPIE Milestone Series, MS171 (SPIE Optical Engineering Press, Bellingham, Wash, 2001).

2. H. J. Coufal, D. Psaltis, and G. T. Sincerbox, eds. Holographic Data Storage (Springer-Verlag, Berlin, 2000).

3. J. M. Kim, B. S. Choi, S. I. Kim, J. M. Kim, H. J. Bjelkhagen, and N. J. Phillips, "Holographic optical elements recorded in silver halide sensitized gelatin emulsions. Part I. Transmission holographic optical elements," Appl. Opt. 40, 622-632 (2001).

4. M. Domingo, I. Arias, and A. García, "Achromatic Fourier processor with holographic optical lenses," Appl. Opt. 40, 22672274 (2001).

5. H. I. Bjelkhagen, Silver Halide Recording Materials for Holography and Their Processing (Springer-Verlag, Berlin, 1993). 
6. D. H. McMahon and A. R. Franklin, "Efficient, high-quality, R-10 bleached holographic diffraction gratings," Appl. Opt. 8, 1927-1929 (1969).

7. J. Upatnieks and C. Leonard, "Diffraction efficiency of bleached, photographically recorded interference patterns," Appl. Opt. 8, 85-89 (1969).

8. P. Hariharan, C. S. Ramanathan, and G. S. Kaushik, "Simplified processing technique for photographic phase holograms," Opt. Commun. 3, 246-247 (1971).

9. J. Crespo, A. Fimia, and J. A. Quintana, "Fixation-free methods in bleached reflection holography," Appl. Opt. 25, 1642 1645 (1986).

10. R. K. Kostuk, "Factorial optimization of bleach constituents for silver halide holograms,” Appl. Opt. 30, 1611-1616 (1991).

11. C. Neipp, I. Pascual, and A. Beléndez, "Effects of overmodulation in fixation-free rehalogenating bleached holograms," Appl. Opt. 40, 3402-3408 (2001).

12. C. Neipp, I. Pascual, and A. Beléndez, "Theoretical and experimental analysis of overmodulation effects in volume holograms recorded on BB-640 emulsions," J. Opt. A: Pure Appl. Opt. 3, 504-513 (2001).

13. R. K. Kostuk and J. W. Goodman, "Refractive index modulation mechanism in bleached silver halide holograms," Appl. Opt. 30, 369-371 (1991).

14. P. Hariharan and C. M. Chidley, "Rehalogenating bleaches for photographic phase holograms: the influence of halide type and concentration on diffraction efficiency and scattering," Appl. Opt. 26, 3895-3898 (1987).

15. P. Hariharan, "Rehalogenating bleaches for photographic phase holograms 3: Ḿechanism of material transfer," Appl. Opt. 29, 2983-2985 (1990).

16. R. L. Van Renesse and F. A. J. Bouts, "Efficiency of bleaching agents for holography," Optik 38, 156-168 (1973).

17. R. L. Van Renesse, "Scattering properties of fine-grained bleached emulsions," Photograph. Sci. Eng. 24, 114-119 (1980).

18. C. Neipp, E. Beronich, I. Pascual, and A. Beléndez, "Fixationfree bleached silver halide transmission holograms recorded on Slavich PFG-01 red sensitive plates," J. Mod. Opt. 48, 16431655 (2001).

19. C. Neipp, I. Pascual, and A. Beléndez, "Bleached silver halide volume holograms recorded on Slavich PFG-01 emulsion: The influence of the developer," J. Mod. Opt. 48, 1479-1494 (2001).

20. H. Kasprzak, N. Sultanova, and H. Pbdbielska, "Nonlinear effects of the recording material on the image quality of a Fourier hologram," J. Opt. Soc. Am. A 4, 843-846 (1987).

21. N. J. Phillips, H. Heyworth, and T. Hare, "On Lippmann's photography,” J. Photogr. Sci. 32, 158-169 (1984).

22. H. D. Thöll, M. Döhmen, and C. G. Stojanoff, "Determination of the mean refractive index and the thickness of dichromated gelatin holographic films using the thin film resonance method," in Holographic Materials, T. J. Trout, ed., Proc. SPIE 2405, 76-87 (1995).

23. P. Hariharan and C. M. Chidley, "Rehalogenating bleaches for photographic phase holograms: spatial frequency effects," Appl. Opt. 27, 3852-3854 (1988).

24. H. Kogelnik, "Coupled wave theory for thick hologram gratings,” Bell Syst. Tech. J. 48, 2909-2947 (1969). 